Case Report

\title{
Usefulness of Stereotactic Radiotherapy Using CyberKnife for Recurrent Lymph Node Metastasis of Differentiated Thyroid Cancer
}

\author{
Joji Kawabe, ${ }^{1}$ Shigeaki Higashiyama, ${ }^{1}$ Mitsuharu Sougawa, ${ }^{2}$ Atsushi Yoshida, ${ }^{1}$ \\ Kohei Kotani, ${ }^{1}$ and Susumu Shiomi ${ }^{1}$ \\ ${ }^{1}$ Department of Nuclear Medicine, Graduate School of Medicine, Osaka City University, Osaka, Japan \\ ${ }^{2}$ CyberKnife Center, Osaka Medical College Mishima-Minami Hospital, Osaka, Japan \\ Correspondence should be addressed to Joji Kawabe; kawabe@med.osaka-cu.ac.jp
}

Received 12 January 2017; Accepted 8 March 2017; Published 15 March 2017

Academic Editor: Thomas Grüning

Copyright (C) 2017 Joji Kawabe et al. This is an open access article distributed under the Creative Commons Attribution License, which permits unrestricted use, distribution, and reproduction in any medium, provided the original work is properly cited.

\begin{abstract}
A woman in her 60 s presented with a recurrent lymph node metastasis from a papillary thyroid carcinoma in the right parapharyngeal space. She had already undergone total thyroidectomy, five resections for cervical lymph node metastases, and right carotid rebuilding. Surgical resection of the current metastasis was impossible. ${ }^{131}$ I-radioiodine therapy (RIT) with 3.7 GBq

${ }^{131}$ I was not effective; therefore, stereotactic radiation therapy (SRT) using a CyberKnife radiotherapy system was scheduled. The prescription dose was $21 \mathrm{~Gy}$, and a dose covering $95 \%$ of the planning target volume (PTV) in three fractions was administered. The PTV was $4,790 \mathrm{~mm}^{3}$. Follow-up magnetic resonance imaging conducted 3 and 12 months after the SRT demonstrated a remarkable and gradual reduction of the recurrent lymph node metastasis in the right parapharyngeal space and no evidence of recurrence. For multidisciplinary therapy of unresectable and/or RIT unresponsive locoregional lymph node metastases and recurrences of DTC, SRT using the CyberKnife system should be considered.
\end{abstract}

\section{Introduction}

The application of external beam radiotherapy (EBRT) as postoperative treatment for differentiated thyroid cancer (DTC) has been controversial in the field of head and neck surgery, as well as in radiation oncology [1-3]. We present a case of recurrent lymph node metastasis of DTC treated effectively with stereotactic radiation therapy (SRT) by using a CyberKnife radiotherapy system (Accuray Inc., Sunnyvale, CA, USA) $[4,5]$. Written informed consent was obtained from the patient for publication of this case report and accompanying images are in accordance with the Code of Ethics of the World Medical Association.

\section{Case Report}

A woman in her 60s presented with a recurrent lymph node metastasis in the right parapharyngeal space from a papillary thyroid carcinoma (Figure 1(a)). After undergoing right lobectomy of the thyroid and right neck dissection 7 years ago, she experienced five episodes of recurrences of cervical lymph node metastases. Resections of the metastases, left thyroid lobectomy, and right carotid rebuilding were performed. At this time, highly abnormal ${ }^{18} \mathrm{~F}$-fluorodeoxyglucose (FDG) uptake was detected in the right parapharyngeal space, which was diagnosed as a recurrent lymph node metastasis (Figure 2(a)). Surgical resection was thought to be difficult due to postsurgical severe changes and carotid rebuilding.

She was referred to our hospital for radioiodine therapy (RIT), which comprised of $3.7 \mathrm{GBq}$ of ${ }^{131} \mathrm{I}$. However, the lymph node metastasis did not take up the ${ }^{131} \mathrm{I}$ (Figure 2(b)). Therefore, the RIT was thought to be ineffective, and EBRT was considered. SRT using the CyberKnife radiotherapy system involves the precise delivery of high dose radiation stereotactically to a target in a small number of fractions. The steep dose fall-off minimizes the radiation to surrounding tissues beyond a few millimeters [4]. 


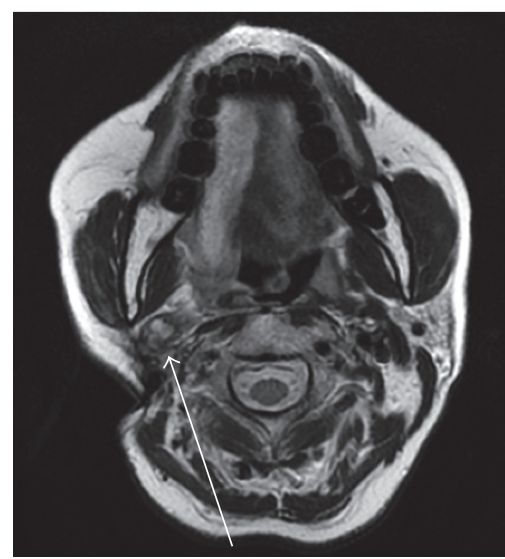

(a)

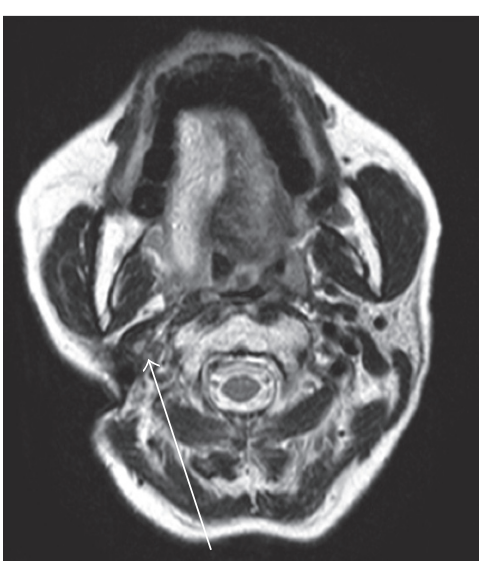

(b)

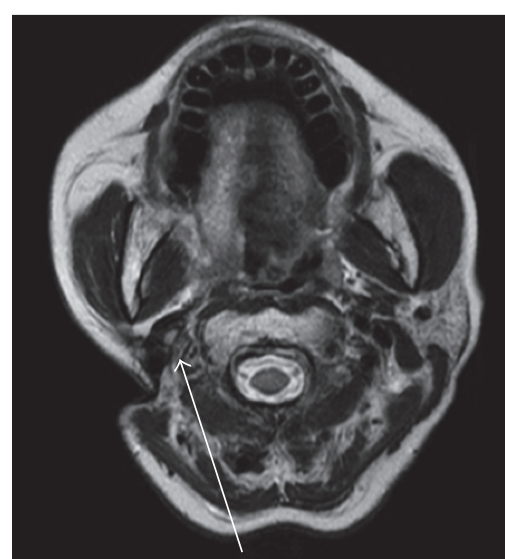

(c)

FIGURE 1: Magnetic resonance T2-weighted imaging findings. (a) Postsurgical status: a recurrent lymph node metastasis from a papillary thyroid carcinoma in the right parapharyngeal space is shown (white arrow). (b) Three months after stereotactic radiation therapy (SRT) using the CyberKnife radiotherapy system, the lymph node metastasis is remarkably reduced. (c) Twelve months after SRT, the reduced lymph node metastasis has not changed. No recurrences were recognized, which indicates therapeutic effectiveness.

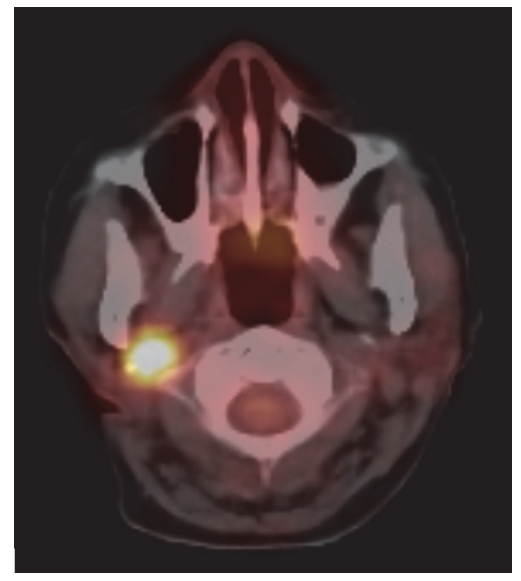

(a)

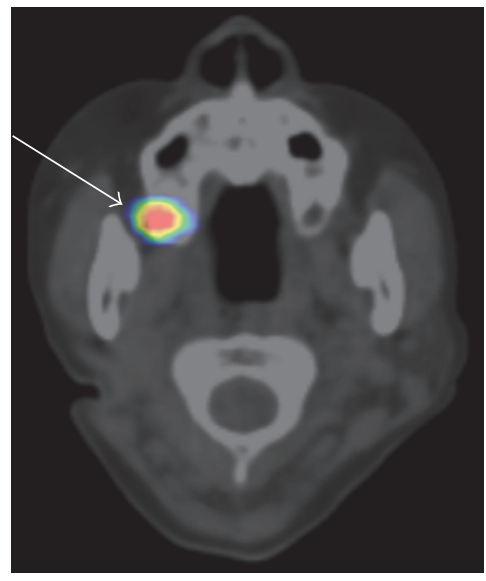

(b)

FIGURE 2: (a) ${ }^{18}$ F-FDG PET/CT image: remarkable abnormal FDG uptake by the lymph node metastasis in the right parapharyngeal space. (b) ${ }^{131}$ I SPECT/CT image obtained 7 days after a ${ }^{131}$ I 3.7 GBq dose of RIT: ${ }^{131}$ I uptake by the lymph node metastasis is not evident. The abnormal distribution in the right maxillary sinus (white arrow) represents ${ }^{131} \mathrm{I}$ secretion into the nasal fluid.

A prescription dose of $21 \mathrm{~Gy}$ and a dose covering $95 \%$ of the planning target volume (PTV) (and the maximum dose of $26.3 \mathrm{~Gy})$ were administered in three fractions at Osaka Medical College Mishima-Minami Hospital CyberKnife Center. The PTV was $4,790 \mathrm{~mm}^{3}$ (Figure 3). The total dose and fractional dose were determined according to the tumor volume and the patient's general condition. Acute adverse events were not observed.

Follow-up magnetic resonance imaging performed 3 and 12 months (Figures 1(b) and 1(c)) after the SRT demonstrated a remarkable and gradual reduction in the recurrent lymph node metastasis in the right parapharyngeal space and no evidence of recurrence.

\section{Discussion}

Generally, postsurgical treatment of DTC patients with metastases involves RIT $[2,3]$, and the use of EBRT has been controversial [1-3]. Ford et al. reported that EBRT requires a total dose of at least $50 \mathrm{~Gy}$ and possibly higher, in addition to RIT, in order to have any impact on local control [1]. Acute (mucositis, dysphagia, skin reactions, and edema) and late (skin fibrosis and tracheal compression) reactions to EBRT have been reported [6-8]. SRT delivers high radiation doses to small lesions with short fractionation schemes under the most stringent conditions, allowing for high dose conformity and sparing of healthy tissue. This should help to overcome 


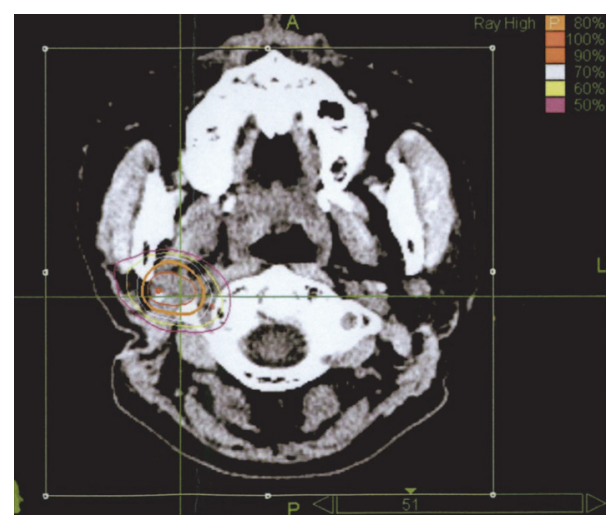

FIgURE 3: Dose distribution for the lymph node metastasis in the right parapharyngeal region. The planning target volume is illustrated by the thin orange line.

the long-term toxicity concerns about conventional EBRT [9].

In our case, surgical resection of the lymph node metastasis in the right parapharyngeal space was impossible. Therapeutic effectiveness of RIT could not be expected. Therefore, we administered SRT using the CyberKnife system, which allowed for a reduction in the lymph node metastasis. A year after the SRT, no recurrence has been detected.

SRT using the CyberKnife system demonstrated good therapeutic performance in our case. The prescribed dose in our case was $21 \mathrm{~Gy}$. Yamazaki et al. reported the prescribed dose to head and neck tumor lesions, including cervical lymph node metastases, ranged from $13 \mathrm{~Gy}$ to $39 \mathrm{~Gy}$, with a median of $30 \mathrm{~Gy}$ [10]. Therefore, a total dose of $21 \mathrm{~Gy}$ was thought to be appropriate.

In 2015, the American Thyroid Association Management Guidelines for Adult Patients with Thyroid Nodules and Differentiated Thyroid Cancer (the ATA guideline) considers using EBRT delivered via modern techniques such as stereotactic radiation for locoregional recurrence that is not surgically resectable. However, as far as we know, no previous report of treating DTC with SRT by using a CyberKnife system has been published.

\section{Conclusion}

For multidisciplinary therapy of unresectable and/or RIT ineffective locoregional lymph node metastases and DTC recurrence, SRT delivered via the CyberKnife system should be considered.

\section{Conflicts of Interest}

The authors declared no conflicts of interest.

\section{References}

[1] D. Ford, S. Giridharan, C. McConkey et al., "External beam radiotherapy in the management of differentiated thyroid cancer," Clinical Oncology, vol. 15, no. 6, pp. 337-341, 2003.
[2] R. T. Kloos, "Papillary thyroid cancer: medical management and follow-up," Current Treatment Options in Oncology, vol. 6, no. 4, pp. 323-338, 2005.

[3] B. R. Haugen, E. K. Alexander, K. C. Bible et al., "2015 American Thyroid Association Management Guidelines for Adult Patients with Thyroid Nodules and Differentiated Thyroid Cancer: The American Thyroid Association Guidelines Task Force on Thyroid Nodules and Differentiated Thyroid Cancer," Thyroid, vol. 26, no. 1, pp. 1-133, 2016.

[4] N. Kodani, H. Yamazaki, T. Tsubokura et al., "Stereotactic body radiation therapy for head and neck tumor: disease control and morbidity outcomes," Journal of Radiation Research, vol. 52, no. 1, pp. 24-31, 2011.

[5] H. Yamazaki, M. Ogita, N. Kodani et al., "Frequency, outcome and prognostic factors of carotid blowout syndrome after hypofractionated re-irradiation of head and neck cancer using CyberKnife: a multi-institutional study," Radiotherapy and Oncology, vol. 107, no. 3, pp. 305-309, 2013.

[6] L. Vini and C. Harmer, "Management of thyroid cancer," Lancet Oncology, vol. 3, no. 7, pp. 407-414, 2002.

[7] N. A. Samaan, P. N. Schultz, R. C. Hickey et al., “The results of various modalities of treatment of well differentiated thyroid carcinoma: a retrospective review of 1599 patients," Journal of Clinical Endocrinology and Metabolism, vol. 75, no. 3, pp. 714720, 1992.

[8] J. Farahati, C. Reiners, M. Stuschke et al., "Differentiated thyroid cancer: impact of adjuvant external radiotherapy in patients with perithyroidal tumor infiltration (stage pT4)," Cancer, vol. 77, no. 1, pp. 172-180, 1996.

[9] I. Veronese, E. De Martin, A. S. Martinotti et al., "Multiinstitutional application of Failure Mode and Effects Analysis (FMEA) to CyberKnife Stereotactic Body Radiation Therapy (SBRT)," Radiation Oncology, vol. 10, no. 1, article 132, 2015.

[10] H. Yamazaki, M. Ogita, K. Himei et al., "Carotid blowout syndrome in pharyngeal cancer patients treated by hypofractionated stereotactic re-irradiation using CyberKnife: a multi-institutional matched-cohort analysis," Radiotherapy and Oncology, vol. 115, no. 1, pp. 67-71, 2015. 


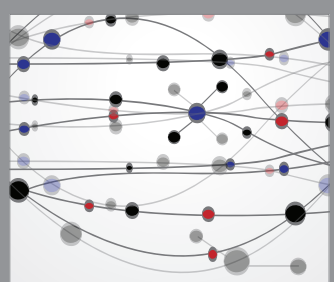

The Scientific World Journal
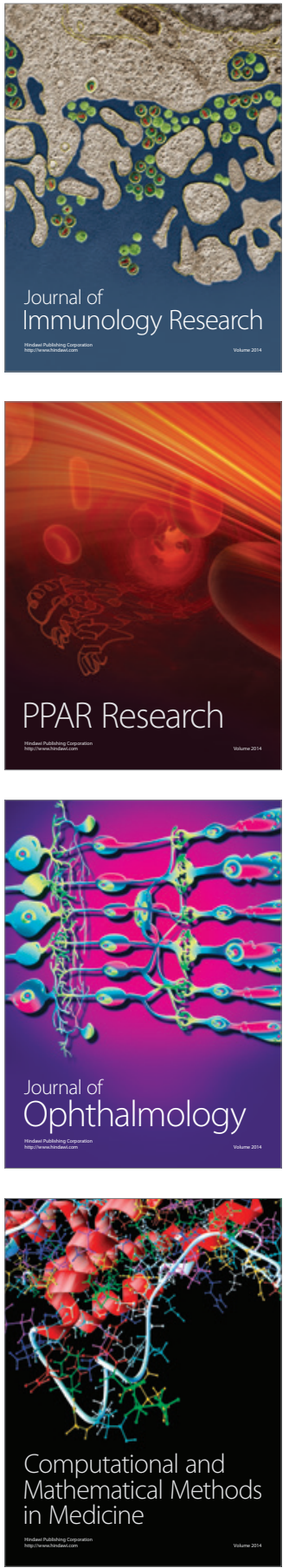

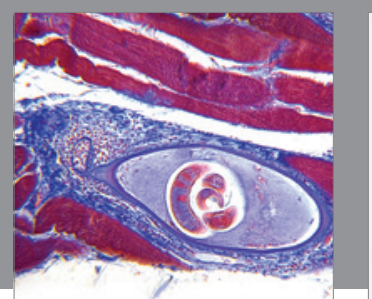

Gastroenterology Research and Practice
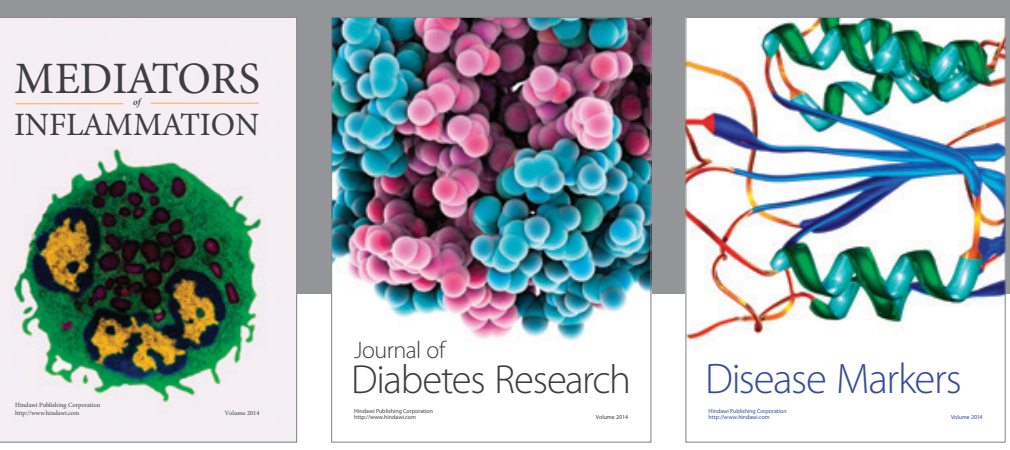

Disease Markers

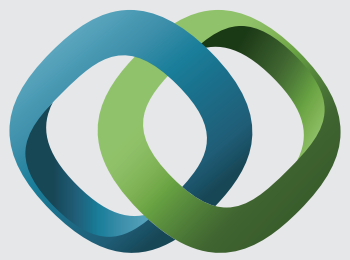

\section{Hindawi}

Submit your manuscripts at

https://www.hindawi.com
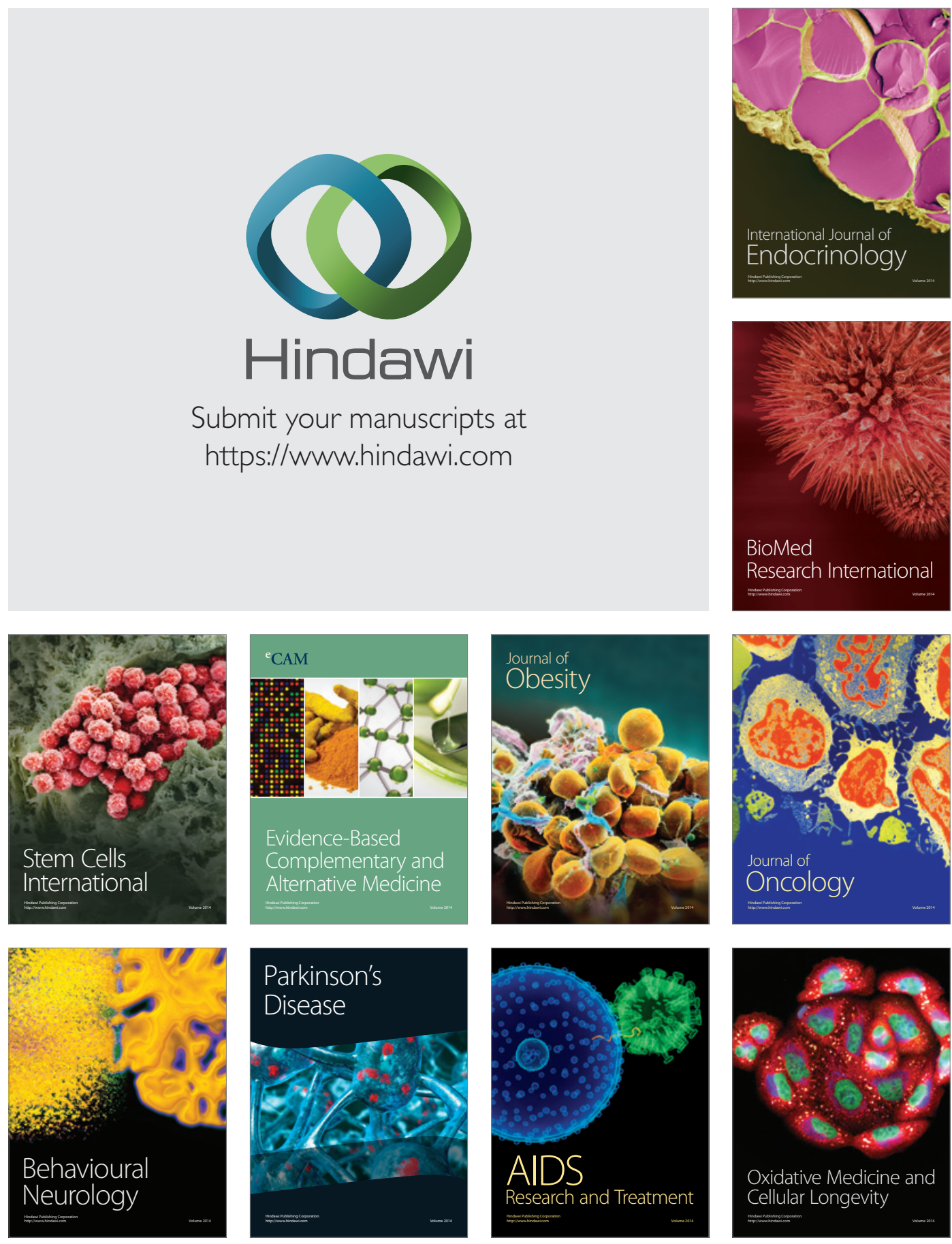